Article

\title{
Effect of Climate and Roasting on Polyphenols and Tocopherols in the Kernels and Skin of Six Hazelnut Cultivars (Corylus avellana L.)
}

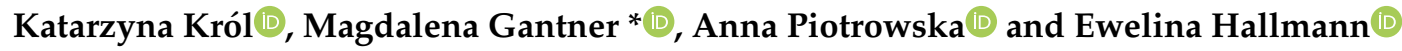 \\ Department of Functional and Organic Food, Institute of Human Nutrition Sciences, \\ Warsaw University of Life Sciences, Nowoursynowska str. 159, 02-776 Warsaw, Poland; \\ katarzyna_krol@sggw.pl (K.K.); anna_piotrowska@sggw.pl (A.P.); ewelina_hallmann@sggw.pl (E.H.) \\ * Correspondence: magdalena_gantner@sggw.pl
}

Received: 8 January 2020; Accepted: 3 February 2020; Published: 6 February 2020

\begin{abstract}
Poland is one of the largest producers of hazelnuts in Europe; however, information about the contents of biologically active compounds in hazelnuts grown in Poland is limited. The aim of this study was to analyze and compare the concentrations of phenolic acids, flavonols, and tocopherols in raw and roasted hazelnut kernels and their skin, in addition to determining the effect of the cultivar and year of cultivation. The experiment was carried out in 2016-2018, and the nuts of six hazelnut cultivars were used for research purposes. Among the detected phenolic acids, $p$-coumaric acid was the most abundant in roasted and unroasted kernels, whereas the apigenin content represented $43-45 \%$ of the total flavonols. The harvest year had a significant effect on the polyphenol content of hazelnuts, whereas roasting had a minor impact. The hazelnut skin contained 168- to 378-fold higher contents of total phenolic compounds than the raw and roasted nuts. Gallic acid was the most abundant phenolic acid in hazelnut skin, contributing $95.0 \%$ of the total polyphenols on average. $\alpha$-Tocopherol was the most abundant tocopherol in the hazelnut cultivars, followed by $\beta+y$-tocopherol and $\delta$-tocopherol in hazelnut cultivars. The statistical analysis showed that roasting and year of cultivation did not affect the tocopherol contents of the investigated samples.
\end{abstract}

Keywords: filbert; Polish hazelnuts; origin; phenolic acid; flavonols; apigenin

\section{Introduction}

Hazelnuts are among the most popular nuts worldwide, mainly cultivated along the coasts of the Black Sea regions of Turkey, as well as in other countries in Europe. Poland is one of the largest producers of hazelnuts in Europe, with production of approximately 5300 tons per year and yield production $1.4 \mathrm{t} \mathrm{ha}^{-1}$ [1]. In Poland the cultivation of only large-fruited, so-called table cultivars, with large, oval nuts has been developed, almost all of which are sold as a raw product for fresh consumption [2].

Polyphenol compounds in nuts are mostly concentrated in the nuts' skin [3]. Several experimental studies have shown the beneficial effects of phenolics in maintaining health and preventing chronic diseases through their antioxidant, anti-inflammatory, cardioprotective and hypolipidemic effects [4-6]. Furthermore, hazelnuts are an excellent source of tocopherols. Among tree nut, hazelnut has been reported to have the highest $\alpha$-tocopherol content [7]. $\alpha$-Tocopherol, which is the tocopherol present at the highest level, exhibits vitamin E activity and is an antioxidant exerting its activity by scavenging free radicals [8-11], and play an important role in the prevention of lipid oxidation, extending shelf-life, and protecting sensory attributes [12].

The processing of hazelnuts, especially roasting, causes changes in the content of phenolic compounds as well as antioxidant activity. Roasting plays the main role in hazelnut processing, leading 
to the improvement of the flavor, color, appearance, and crispiness of the nuts [13]. Hazelnuts are also roasted to remove their skin, which presents a bitter taste after roasting and lowers their value. The hazelnut skin represents approximately $2.5 \%$ of the total hazelnut kernel weight [14]; as a byproduct it is a rich source of natural polyphenol compounds and fiber, and represents a valuable cheap waste material containing natural bioactive compounds [15]. Therefore, hazelnut skin can be used for food enrichment as a potential source of antioxidants and fiber, as well as a pharmaceutical or coloring agent [16-19].

In recent years, various studies have highlighted the polyphenol content of hazelnut and hazelnut skin $[17,20,21]$. However, studies related to the polyphenol content of the hazelnut seed coat have mostly involved quantitative systems [18,21], and most of these studies concerned a single cultivar and only one year of experimentation. Furthermore, the available data regarding the seasonal variability of biologically active compounds in hazelnuts grown in Poland are limited. Therefore, the aims of this work were to move the research into detailed descriptions of the polyphenols and tocopherols of hazelnuts grown under Polish climatic conditions forward, as well as to determine the effect of the cultivar and year of cultivation. This information may contribute to a better estimation of flavonoid intake and broaden the understanding of cultivar-specific flavonoid and phenolic synthesis in hazelnuts.

\section{Materials and Methods}

\subsection{Plant Material}

The experiment was carried out in 2016-2018. Nuts of six hazelnut cultivars ('Barcelonski', 'Katalonski', 'Webba Cenny', 'Cosford', 'Olbrzym z Halle', and 'Notthingamski') were cultivated in an orchard located in southeastern Poland (Lubelskie, Końskowola, $51^{\circ} 25^{\prime} \mathrm{N} 22^{\circ} 03^{\prime}$ E). All of the nuts were collected at technological maturity in September of each year. The weather conditions in the orchard are recorded by a meteorological station (Lublin-Radawiec no. 351220495) of Polish Institute of Meteorology and Water Management [22]. The chosen 2.5 ha plantation is flat. Hazel trees grow at a in spacing of $3.0 \times 4.5 \mathrm{~m}$. The plants were trained as vertical axis $3 \mathrm{~m}$ high, and the plants are regularly pruned to illuminate individual branches well. All of the selected cultivars are listed in the Polish National List of Fruit Plant Varieties 2019 [23]. Nut samples totaling $5 \mathrm{~kg}$ per cultivar/year were handpicked from the soil, shortly after dropping on their own to the ground, two or three times, depending on the cultivar. The time from the fall of the nuts to their harvest did not exceed $48 \mathrm{~h}$. After harvest, they were dried to $6 \%$ kernel humidity according to the current postharvest treatments applied in commercial production and then stored at $20^{\circ} \mathrm{C}$. The nuts were cracked by hand, and then $2.5 \mathrm{~kg}$ of the nuts of each cultivar was roasted at $130^{\circ} \mathrm{C}$ for $30 \mathrm{~min}$ in a universal dryer with electronic temperature control (SUP-200G, Wamed, Poland). These conditions were set according to the range of conditions commonly used in the hazelnut industry. After roasting, the hazelnuts were peeled manually and placed in plastic bags. Then, the samples were stored in a cool, dry and dark place (at $4{ }^{\circ} \mathrm{C} \pm 1$ ) to maintain their chemical characteristics until they were analyzed.

\subsection{Phenolic Acid and Flavonol Separation and Identification}

Polyphenols were measured using high-performance liquid chromatography (HPLC). Before analysis, the hazelnut samples (raw or roasted without skin) were grounded with a mechanical coffee mill (Bosh, Germany, MKM6003). One gram of milled raw or roasted hazelnut kernels was weighed (Radwag WPX 1500, Radom, Poland) and extracted in $100 \mathrm{~mL}$ deionized water, followed by purification with Carrez solutions ( $5 \mathrm{~mL}$ each) to remove precipitated proteins. After $10 \mathrm{~min}$, the extracts were filtered through paper filter into a $250 \mathrm{~mL}$ beaker. Carrez solutions (I and II) were prepared according to the method described by Wang et al. [10]. The method of hazelnuts extractions was performed according to Tas and Gokmen [4] with slight modification. One gram of roasted hazelnut skin was extracted in $5 \mathrm{~mL}$ of $80 \%$ methanol with shaking on a Micro-Shaker $326 \mathrm{M}$ (Poland). The samples were 
incubated in an ultrasonic bath $\left(10 \mathrm{~min}, 30^{\circ} \mathrm{C}, 550 \mathrm{~Hz}\right)$. After $10 \mathrm{~min}$, samples were centrifuged $(10 \mathrm{~min}$, $6000 \mathrm{RPM}, 0{ }^{\circ} \mathrm{C}$ ). A $1 \mathrm{~mL}$ aliquot of the extract supernatant was transferred to vials and analyzed. To determine polyphenols, the HPLC- set-ups up was prepared, consisting of two LC-20AD pumps, a CMB-20A system controller, a SIL-20AC autosampler, an ultraviolet-visible SPD-20AV detector, a CTD-20AC oven, and a Phenomenex Fusion-RP 80A column $(250 \times 4.60 \mathrm{~mm}$; column particle size $4 \mu \mathrm{m})$, from Shimadzu (Shimadzu, Tokyo, Japan). Two gradient phases were used: phase A-10\% (v:v) acetonitrile and ultrapure water; phase B-55\% (v:v) acetonitrile and ultra-pure water. The phases were acidified by $85 \%{ }_{0}{ }_{-3} \mathrm{PO}_{4}(\mathrm{pH} 3.0)$. The analysis time was $38 \mathrm{~min}$. The phase-time program was as follows: $1.00-22.99 \mathrm{~min} 95 \%$ phase $\mathrm{A}$ and $5 \%$ phase $\mathrm{B}, 23.00-27.99 \mathrm{~min} 50 \%$ phase $\mathrm{A}$ and $50 \%$ phase $\mathrm{B}$, $28.00-28.99 \mathrm{~min} 80 \%$ phase $A$ and $20 \%$ phase $B$, and $29.00-38.00 \mathrm{~min} 95 \%$ phase $A$ and $5 \%$ phase $B$. Two wavelengths of $250 \mathrm{~nm}$ (flavonol) and $370 \mathrm{~nm}$ (phenolic acid) were applied. The phenolic compounds were identified on the basis of external standards (Sigma-Aldrich, Poznan, Poland) with purity of 99.5\%. Standard curves for phenolic compounds are presented in the Appendix A Figures A1 and A2.

\subsection{Tocopherol Analysis}

The kernels of the hazelnuts were sliced and oil was extracted via cold pressing at low temperature in a continuous screw press, with a capacity of $40 \mathrm{~kg} / \mathrm{h}$ of nuts, equipped with an engine of $460 \mathrm{~W}$ (Oleum, KT-OPM, Hangzhou, Zhejiang, China). The concentration and extraction of vitamin E was determined in oil from raw and roasted kernels according to method PN-EN ISO 9936:2016 [24] reversed-phase high-pressure liquid chromatography (HPLC-FLD) system with a RF-10AXL fluorescence detector (Shimadzu, Kyoto, Japan), equipped with 5C18-MS-II column $(150 \times 4.6 \mathrm{~mm}$ I.D., column particle size $5 \mu \mathrm{m}$ Cosmosil). The mobile phase was methanol $(100 \%)$ at a flow rate of $1.0 \mathrm{~mL} \mathrm{~min}^{-1}$. The excitation wavelength was $295 \mathrm{~nm}$, and the emission was $330 \mathrm{~nm}$. The method of tocopherol determination in food samples was accredited by the Polish Centre for Accreditation (accreditation number AB 079). The detection range of the method was from $0.1 \mathrm{mg}$ tocopherols in $100 \mathrm{~g}$ of analyzed oil to $30,000 \mathrm{mg}$ tocopherols in $100 \mathrm{~g}$ of analyzed oil. The concentrations of $\alpha$-tocopherol, $\delta$-tocopherol, the sum of $\beta$ - and $y$-tocopherols as well as the sum of total tocopherols as the sum of each tocopherol were

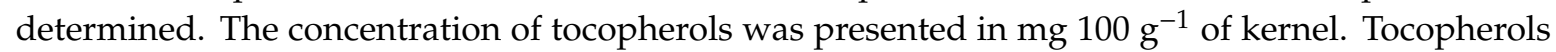
were identified by their retention times as compared to external standards obtained from Fluka and Sigma-Aldrich (Poland) and ran at the same conditions.

\subsection{Statistical Analysis}

The obtained results were statistically analyzed with XLSTATS by Addinsoft 2019.3.2. version software (Boston, Massachusetts, USA). Two-way ANOVA analysis was conducted followed by Tukey's multiple comparisons. Results were expressed as mean \pm SD of three independent experiments $(n=3)$. The factors within the experiment were the cultivars of hazelnuts, years of cultivation and the roasting process. A lack of significant differences $(p>0.05)$ is indicated in the tables as not significant (N.S). Different letters within a row or column indicate significant differences at the $p<0.05$ level. Multivariate statistical analysis was performed using principal component analysis (PCA) to obtain a clearer visualization between the identified polyphenols, tocopherols and hazelnut cultivars using the mean value.

\section{Results}

\subsection{Weather Conditions}

Detailed information on the climate conditions (average minimum and maximum temperature, number of hours of sunshine per day and rainfall per month) is shown in Figure 1. Among the study years, 2018 was characterized by the highest maximum temperature followed by 2016 and 2017, whereas the lowest minimum temperature was observed in 2016, followed by 2017 and 2018. The highest rainfall was observed in 2017, whereas the lowest rainfall was observed in 2018. The greatest 
number of sunshine hours per month occurred in 2017, whereas in 2018, the number was lower but not significantly so.

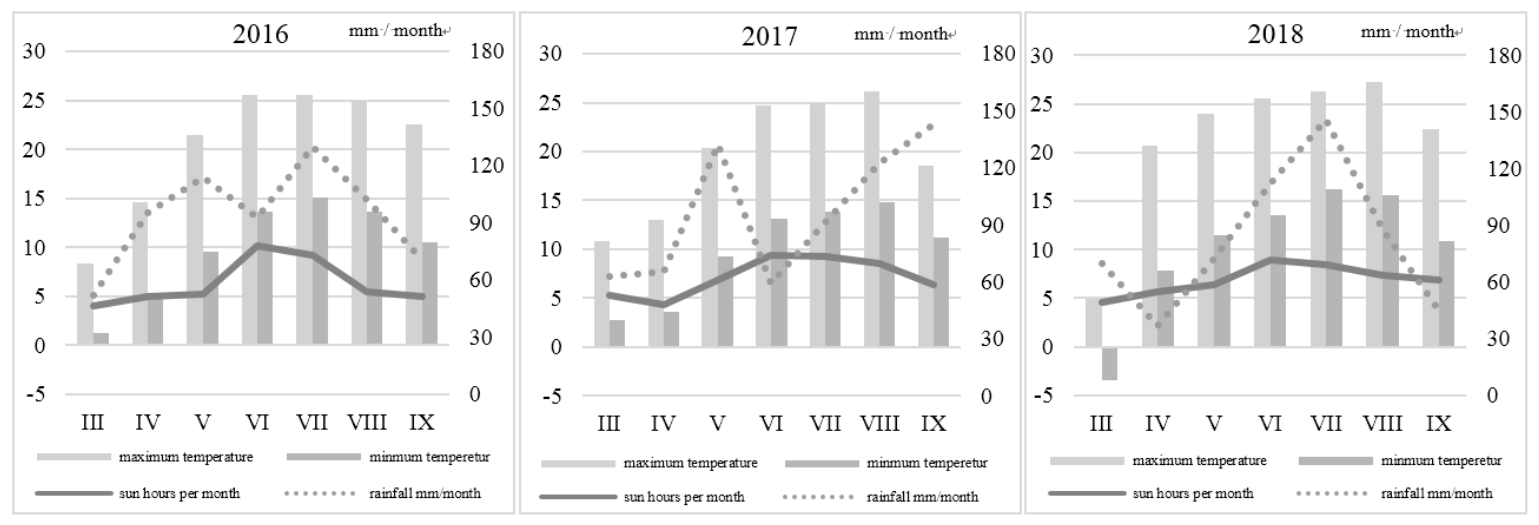

Figure 1. Weather conditions in the time-period 2016-2018 during hazelnut kernel development.

\subsection{Chemical Characteristics}

\subsubsection{Polyphenol Content}

Table 1 summarizes the composition and average content of polyphenolic compounds in raw hazelnut kernels, roasted hazelnut kernels without the skin and the skin alone in 2016-2018. HPLC identification of individual polyphenolic compounds of kernels showed the presence of 4 phenolic acids (gallic acid, caffeic acid, $p$-coumaric acid, ferulic acid) and 6 flavonols (quercetin, catechin, epigallocatechin, quercetin-3-O-rutinoside, kaempferol-3-O-glucoside, apigenin). Gallic acid is a hydroxylated derivative of benzoic acid, whereas caffeic acid, $p$-coumaric acid, and ferulic acid are cinnamic acid derivatives. Overall, the highest total polyphenol content in raw nut kernels was detected in 2018, followed by 2017 and 2016. However, the total phenolic acid level was the highest in 2018, followed by 2016 and 2017, whereas the total flavonol contents were highest in 2017, followed by 2018 and 2016. The results showed that the main constituent of the total polyphenol compounds present in raw and roasted hazelnut kernels was phenolic acids, accounting for between $60-62 \%$ of the total polyphenols. Among the detected phenolic acids, $p$-coumaric acid was the most abundant in roasted and unroasted kernels, followed by caffeic, gallic and ferulic acid, which showed mean values of $11.59,4.78,3.96$ and $2.16 \mathrm{mg} \mathrm{kg}^{-1} \mathrm{fw}$, respectively. The order of the flavonols in the unroasted and roasted hazelnuts according to their content was as follows: apigenin $>$ kaempferol-3-O-glucoside $>$ quercetin $>$ catechin $>$ epigallocatechin $>$ quercetin-3-O-rutinoside. The apigenin content was among the most stable between the three years of analysis, with an average content of $6.08 \mathrm{mg} \mathrm{kg}^{-1} \mathrm{fw}$, representing $43-45 \%$ of the total flavonols. Quercetin-3-O-rutinoside and epigallocatechin represented approximently $3 \%$ of the total polyphenols. The statistical analysis showed that the year of cultivation had a significant effect on the $p$-coumaric, ferulic, quercetin-3-O-rutinoside, kaempferol-3-O-glucoside, and epigallocatechin contents; therefore, the values increased or decreased significantly in different ways between the 3 years of the experiment. The largest increases during the 3 years were observed for $p$-coumaric acid and kaempferol-3-O-glucoside, whereas the largest decrease was observed for ferulic acid. Furthermore, skin removal after roasting process led to a significant reduction in the concentration of gallic acid $(p<0.0001)$, wheras an increase $(p=0.035)$ in ferulic acid was observed, suggesting that thermal processing had a minor impact on the polyphenol content of hazelnut kernels. The hazelnut skin contained 168- to 378- fold higher contents of total phenolic compounds than raw and roasted nuts, respectively. Gallic acid was the most abundant phenolic acid, contributing $95.0 \%$ of the total polyphenolic compounds, and $98.2 \%$ of the phenolic acids on average. Year of cultivation mainly influenced the flavonoid contents, and significant differences were observed for kaempferol-3-O-glucoside, catechin, epigallocatechin, and quercetin-3-O-rutinoside. 
Table 1. Chemical composition of raw roasted hazelnut kernels and hazelnut skin over 3 years (2016-2018) (mean of all cultivars \pm SD; mg kg ${ }^{-1}$ fresh weight).

\begin{tabular}{|c|c|c|c|c|c|c|c|c|c|c|c|c|c|c|}
\hline & & $\begin{array}{c}\text { Total } \\
\text { Polyphenols }\end{array}$ & $\begin{array}{c}\text { Total } \\
\text { Phenolic } \\
\text { Acid }\end{array}$ & $\begin{array}{l}\text { Gallic } \\
\text { Acid }\end{array}$ & $\begin{array}{l}\text { Caffeic } \\
\text { Acid }\end{array}$ & $\begin{array}{l}p \text {-Coumarc } \\
\text { Acid }\end{array}$ & $\begin{array}{l}\text { Ferulic } \\
\text { Acid }\end{array}$ & $\begin{array}{c}\text { Total } \\
\text { Flavonoids }\end{array}$ & $\begin{array}{l}\text { Quercetin-3- } \\
\text { ORutinoside }\end{array}$ & Catechin & $\begin{array}{l}\text { Epigallo- } \\
\text { Catechin }\end{array}$ & $\begin{array}{c}\text { Kaempferol- } \\
\text { 3-O- } \\
\text { Glucoside }\end{array}$ & Quercetin & Apigenin \\
\hline \multirow{5}{*}{$\underset{\nwarrow}{\approx}$} & 2016 & $\begin{array}{l}34.97 \pm \\
2.43^{a *}\end{array}$ & $\begin{array}{c}22.00 \pm \\
1.51^{\mathrm{a}}\end{array}$ & $\begin{array}{l}3.99 \pm \\
0.15^{\mathrm{a}}\end{array}$ & $\begin{array}{l}4.82 \pm \\
0.32^{\mathrm{a}}\end{array}$ & $\begin{array}{c}10.35 \pm \\
0.56^{\mathrm{b}}\end{array}$ & $\begin{array}{l}2.23 \pm \\
0.19^{\mathrm{a}}\end{array}$ & $\begin{array}{c}13.40 \pm \\
0.04^{\mathrm{b}}\end{array}$ & $\begin{array}{l}0.47 \pm \\
0.11 \mathrm{ab}\end{array}$ & $\begin{array}{l}1.57 \pm \\
0.03^{\mathrm{a}}\end{array}$ & $\begin{array}{l}0.62 \pm \\
0.06^{b}\end{array}$ & $\begin{array}{l}2.49 \pm \\
0.04^{\mathrm{b}}\end{array}$ & $\begin{array}{l}2.16 \pm \\
0.03^{a}\end{array}$ & $\begin{array}{l}6.09 \pm \\
0.01^{\mathrm{a}}\end{array}$ \\
\hline & 2017 & $36.62 \pm$ & $21.56 \pm$ & $4.06 \pm$ & $4.97 \pm$ & $11.02 \pm$ & $2.07 \pm$ & $14.61 \pm$ & $1.23 \pm$ & $1.60 \pm$ & $0.93 \pm$ & $2.87 \pm$ & $1.88 \pm$ & $6.09 \pm$ \\
\hline & 2017 & $1.12^{\mathrm{a}}$ & $2.10^{\mathrm{a}}$ & $0.24^{\mathrm{a}}$ & $0.46^{\mathrm{a}}$ & $1.38^{\mathrm{ab}}$ & $0.12^{a b}$ & $0.05^{\mathrm{a}}$ & $0.16^{\mathrm{a}}$ & $0.05^{\mathrm{a}}$ & $0.05^{\mathrm{a}}$ & $0.05^{\mathrm{a}}$ & $0.05^{\mathrm{a}}$ & $0.00^{\mathrm{a}}$ \\
\hline & 2018 & $37.21 \pm$ & $\begin{array}{r}23.29 \pm \\
2.0\end{array}$ & $4.73 \pm$ & $\begin{array}{l}4.29 \pm \\
0.27 a\end{array}$ & $12.24 \pm$ & $2.02 \pm$ & $13.91 \pm$ & $0.37 \pm$ & $1.58 \pm$ & $0.91 \pm$ & $2.91 \pm$ & $2.04 \pm$ & $6.08 \pm$ \\
\hline & $p$-value year & $\begin{array}{l}3.14^{\mathrm{a}} \\
\text { N.S ** }\end{array}$ & $\begin{array}{l}3.21^{\mathrm{a}} \\
\mathrm{N} . \mathrm{S}\end{array}$ & $\begin{array}{c}1.32^{\mathrm{a}} \\
\text { N.S }\end{array}$ & $\begin{array}{l}0.27^{\mathrm{a}} \\
\text { N.S }\end{array}$ & $\begin{array}{c}3.21^{\mathrm{a}} \\
<0.0001\end{array}$ & $\begin{array}{l}0.23^{\mathrm{b}} \\
0.062\end{array}$ & $\begin{array}{l}0.03^{\mathrm{ab}} \\
<0.0001\end{array}$ & $\begin{array}{c}0.06^{\mathrm{b}} \\
<0.0001\end{array}$ & $\begin{array}{l}0.05^{\mathrm{a}} \\
\text { N.S }\end{array}$ & $\begin{array}{c}0.04^{\mathrm{a}} \\
<0.0001\end{array}$ & $\begin{array}{l}0.06^{\mathrm{a}} \\
0.052\end{array}$ & $\begin{array}{c}0.05^{\mathrm{a}} \\
<0.0001\end{array}$ & $\begin{array}{l}0.01^{\mathrm{a}} \\
\text { N.S }\end{array}$ \\
\hline \multirow{6}{*}{ 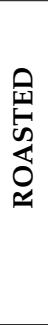 } & 2016 & $\begin{array}{c}35.56 \pm \\
2.50^{\mathrm{a}}\end{array}$ & $\begin{array}{l}22.24 \pm \\
2.15^{\mathrm{a}}\end{array}$ & $\begin{array}{l}3.48 \pm \\
0.27^{\mathrm{a}}\end{array}$ & $\begin{array}{l}5.28 \pm \\
0.28^{a}\end{array}$ & $\begin{array}{l}11.04 \pm \\
0.43^{\mathrm{a}}\end{array}$ & $\begin{array}{l}2.44 \pm \\
0.42^{\mathrm{a}}\end{array}$ & $\begin{array}{c}13.31 \pm \\
0.80^{\mathrm{b}}\end{array}$ & $\begin{array}{l}0.32 \pm \\
0.17 \mathrm{ab}\end{array}$ & $\begin{array}{l}1.57 \pm \\
0.02^{a}\end{array}$ & $\begin{array}{l}0.60 \pm \\
0.12^{b}\end{array}$ & $\begin{array}{l}2.49 \pm \\
0.59^{b}\end{array}$ & $\begin{array}{l}2.25 \pm \\
0.01^{\mathrm{a}}\end{array}$ & $\begin{array}{l}6.07 \pm \\
0.03^{a}\end{array}$ \\
\hline & 2017 & $\begin{array}{c}37.04 \pm \\
4.10^{\mathrm{a}}\end{array}$ & $\begin{array}{c}22.61 \pm \\
2.54^{\mathrm{a}}\end{array}$ & $\begin{array}{l}3.38 \pm \\
0.23^{a}\end{array}$ & $\begin{array}{l}4.95 \pm \\
0.43^{a}\end{array}$ & $\begin{array}{c}12.06 \pm \\
2.11^{\mathrm{a}}\end{array}$ & $\begin{array}{l}2.20 \pm \\
0.17^{\mathrm{b}}\end{array}$ & $\begin{array}{c}14.42 \pm \\
1.91^{\mathrm{a}}\end{array}$ & $\begin{array}{l}1.00 \pm \\
0.21^{\mathrm{a}}\end{array}$ & $\begin{array}{l}1.60 \pm \\
0.04^{a}\end{array}$ & $\begin{array}{l}0.91 \pm \\
0.20^{a}\end{array}$ & $\begin{array}{l}2.84 \pm \\
0.50^{a}\end{array}$ & $\begin{array}{l}1.97 \pm \\
0.09^{a}\end{array}$ & $\begin{array}{l}6.08 \pm \\
0.01^{a}\end{array}$ \\
\hline & 2018 & $36.90 \pm$ & $23.07 \pm$ & $3.80 \pm$ & $4.38 \pm$ & $12.83 \pm$ & $2.04 \pm$ & $13.83 \pm$ & $0.29 \pm$ & $1.58 \pm$ & $0.87 \pm$ & $2.87 \pm$ & $2.11 \pm$ & $6.08 \pm$ \\
\hline & 2010 & $3.20^{\mathrm{a}}$ & $2.99^{\mathrm{a}}$ & $0.94^{\mathrm{a}}$ & $0.21^{\mathrm{a}}$ & $3.21^{\mathrm{a}}$ & $0.15^{\mathrm{b}}$ & $0.83^{\mathrm{ab}}$ & $0.14^{\mathrm{b}}$ & $0.04^{\mathrm{a}}$ & $0.26^{\mathrm{a}}$ & $0.84^{\mathrm{a}}$ & $0.18^{\mathrm{a}}$ & $0.05^{\mathrm{a}}$ \\
\hline & $p$-value year & N.S & N.S & N.S & N.S & N.S & 0.029 & $<0.0001$ & $<0.0001$ & 0.048 & 0.033 & 0.036 & $<0.0001$ & N.S \\
\hline & $\begin{array}{l}p \text {-value } \\
\text { roasting }\end{array}$ & N.S & N.S & $<0.0001$ & N.S & N.S & 0.035 & N.S & N.S & N.S & N.S & N.S & N.S & N.S \\
\hline \multirow{5}{*}{$\frac{Z}{w}$} & 2016 & $\begin{array}{c}9718.12 \pm \\
1329^{\mathrm{a}}\end{array}$ & $\begin{array}{r}9421.83 \\
\pm 1357^{\mathrm{a}}\end{array}$ & $\begin{array}{l}9239.23 \\
\pm 399^{\text {a }}\end{array}$ & $\begin{array}{c}142.64 \pm \\
13.2^{\mathrm{a}}\end{array}$ & $\begin{array}{c}23.63 \pm \\
2.43^{\mathrm{a}}\end{array}$ & $\begin{array}{c}15.63 \pm \\
3.73^{\mathrm{a}}\end{array}$ & $\begin{array}{c}296.29 \pm \\
24.61^{\mathrm{b}}\end{array}$ & $\begin{array}{l}20.94 \pm \\
1.28^{\mathrm{ab}}\end{array}$ & $\begin{array}{c}151.34 \pm \\
10.83^{\mathrm{b}}\end{array}$ & $\begin{array}{c}10.96 \pm \\
2.48^{\mathrm{b}}\end{array}$ & $\begin{array}{l}3.85 \pm \\
0.34 \mathrm{~b}\end{array}$ & $\begin{array}{c}23.24 \pm \\
9.27^{\mathrm{a}}\end{array}$ & $\begin{array}{c}85.95 \pm \\
6.59^{\mathrm{a}}\end{array}$ \\
\hline & 2017 & $\begin{array}{c}9304.34 \pm \\
1693 \text { a }\end{array}$ & $\begin{array}{r}8889.44 \\
\pm 1687 \mathrm{a}\end{array}$ & $\begin{array}{r}9051.88 \\
\pm 1357^{\text {a }}\end{array}$ & $\begin{array}{c}122.94 \pm \\
15.3^{\mathrm{a}}\end{array}$ & $\begin{array}{c}28.02 \pm \\
2.08^{a}\end{array}$ & $\begin{array}{c}13.28 \pm \\
4.01^{\mathrm{b}}\end{array}$ & $\begin{array}{l}414.32 \pm \\
10279 \mathrm{a}\end{array}$ & $\begin{array}{c}30.19 \pm \\
6.43^{\mathrm{a}}\end{array}$ & $\begin{array}{l}235.67 \pm \\
114.85 a\end{array}$ & $\begin{array}{c}18.44 \pm \\
4.20^{\mathrm{a}}\end{array}$ & $\begin{array}{c}16.33 \pm \\
781^{\mathrm{a}}\end{array}$ & $17.77 \pm$ & $\begin{array}{c}95.90 \pm \\
7.29^{\mathrm{a}}\end{array}$ \\
\hline & & $9487.94 \pm$ & 9183.33 & 8725.63 & $88.51 \pm$ & $31.32 \pm$ & $11.60 \pm$ & $304.05 \pm$ & $20.31 \pm$ & $142.79 \pm$ & $18.08 \pm$ & $17.36 \pm$ & $20.59 \pm$ & $84.89 \pm$ \\
\hline & 2018 & $484^{\mathrm{a}}$ & $\pm 469^{\mathrm{a}}$ & $\pm 1705^{\mathrm{a}}$ & $23.56^{\mathrm{b}}$ & $8.77^{\mathrm{a}}$ & $2.02^{b}$ & $96.6^{\mathrm{b}}$ & $3.07^{\mathrm{b}}$ & $88.45^{b}$ & $3.59^{\mathrm{a}}$ & $2.10^{\mathrm{a}}$ & $2.89^{\mathrm{a}}$ & $8.74^{\mathrm{a}}$ \\
\hline & $p$-value year & N.S & N.S & N.S & $<0.0001$ & N.S & $<0.0001$ & $<0.0001$ & $<0.0001$ & $<0.0001$ & $<0.0001$ & $<0.0001$ & N.S & N.S \\
\hline
\end{tabular}

${ }^{*}$ Values followed by different small letters $(\mathrm{a}-\mathrm{b})$ in the same row are significantly different $(p<0.05) .{ }^{* *}$ N.S-not significant statistically. 
The significant effect on polyphenol content varied differently among cultivars and year of the study (Table 2). In 2016 no significant effect of cultivars was observed for $p$-coumaric acid, catechin and apigenin; in 2017 for apigenin; or in 2018 for caffeic acid, catechin, kaempferol-3-O-glucoside, and apigenin. The order of the contents total polyphenolic compounds in 2016-2018 in hazelnut cultivars was as follows: $p$-coumaric acid $>$ apigenin $>$ caffeic acid $>$ gallic acid $>$ kaempferol-3-O-glucoside $>$ ferulic acid $>$ quercetin $>$ catechin $>$ epigallocatechin $>$ quercetin-3-O-rutinoside. Over three seasons, the lowest content of total polyphenols and phenolic acids was detected in the 'Notthingamski' cultivar (32.15-35.50 mg kg-1), whereas the highest in 2016 was observed for 'Olbrzym z Halle', in 2017 for 'Barceloński', in 2018 for 'Webba Cenny', at 39.08, 39.85, $43.30 \mathrm{mg} \mathrm{kg}$ ', respectively. The main component among the polyphenol compounds was $p$-coumaric acid, which varied from 9.88 to $19.61 \mathrm{mg} \mathrm{kg}^{-1}$ and the 'Webba Cenny' cultivar in 2018 showed a 38\% to 50\% higher content than the other cultivars. The content of gallic acid in the 'Katalonski' cultivar in $2018\left(8.43 \mathrm{mg} \mathrm{kg}^{-1}\right)$ and caffeic acid in the 'Olbrzym z Halle' cultivar $\left(9.13 \mathrm{mg} \mathrm{kg}^{-1}\right)$ were found to be higher than in the other cultivars in current study among 3 years of the experiment. The highest total flavonoids content was observed in the 'Cosford' cultivar in 2017 (18.89 $\left.\mathrm{mg} \mathrm{kg}^{-1}\right)$, which was related to the highest quercetin-3-O-rutinoside content $\left(4.87 \mathrm{mg} \mathrm{kg}^{-1}\right)$ among six investigated cultivars. Apigenin was found to be a major flavonoid in the hazelnut cultivars and its content varied from 6.03 to $6.11 \mathrm{mg} \mathrm{kg}^{-1}$, overall. The accumulation of kaempferol-3-O-glucoside showed a slight (but significant) difference between the investigated cultivars among 3years, and varied from $2.48-3.25 \mathrm{mg} \mathrm{kg}^{-1}$. Epigallocatechin in 2016 and 2018 showed the highest content in 'Barceloński' cultivar, 0.78 and $1.45 \mathrm{mg} \mathrm{kg}^{-1}$, respectively. The content of catechin was statistically significant, however, the differences between cultivars were very small, ranging between from $1.55-1.68 \mathrm{mg} \mathrm{kg}^{-1}$. 
Table 2. Chemical composition of six unroasted hazelnut cultivars in 2016-2018 (mean \pm SD) $\mathrm{mg} \mathrm{kg}^{-1}$ of fresh weight.

\begin{tabular}{|c|c|c|c|c|c|c|c|c|c|c|c|c|c|}
\hline & $\begin{array}{c}\text { Total } \\
\text { Polyphenols }\end{array}$ & $\begin{array}{c}\text { Total } \\
\text { Phenolic } \\
\text { Acid }\end{array}$ & $\begin{array}{l}\text { Gallic } \\
\text { Acid }\end{array}$ & $\begin{array}{c}\text { Caffeic } \\
\text { Acid }\end{array}$ & $\begin{array}{c}p \text {-Coumarc } \\
\text { Acid }\end{array}$ & $\begin{array}{c}\text { Ferulic } \\
\text { Acid }\end{array}$ & $\begin{array}{c}\text { Total } \\
\text { Flavonoids }\end{array}$ & $\begin{array}{c}\text { Quercetin- } \\
3-O- \\
\text { Rutinoside }\end{array}$ & Catechin & $\begin{array}{l}\text { Epigallo- } \\
\text { Catechin }\end{array}$ & $\begin{array}{c}\text { Kaempferol- } \\
\text { 3-O- } \\
\text { Glucoside }\end{array}$ & Quercetin & Apigenin \\
\hline \multicolumn{14}{|l|}{2016} \\
\hline Barceloński & $\begin{array}{l}36.21 \pm \\
0.51 \mathrm{~b} *\end{array}$ & $\begin{array}{l}21.31 \pm \\
0.79 \mathrm{bc}\end{array}$ & $\begin{array}{l}4.55 \pm \\
0.07^{a}\end{array}$ & $\begin{array}{l}4.40 \pm \\
0.03^{b}\end{array}$ & $\begin{array}{c}10.47 \pm \\
0.27^{\mathrm{a}}\end{array}$ & $\begin{array}{l}1.89 \pm \\
0.03^{c}\end{array}$ & $\begin{array}{c}14.91 \pm \\
0.02^{\mathrm{a}}\end{array}$ & $\begin{array}{l}1.24 \pm \\
0.07^{\mathrm{a}}\end{array}$ & $\begin{array}{l}1.55 \pm \\
0.03^{a}\end{array}$ & $\begin{array}{l}0.78 \pm \\
0.02^{a}\end{array}$ & $\begin{array}{l}2.48 \pm \\
0.09^{c}\end{array}$ & $\begin{array}{l}2.75 \pm \\
0.00^{a}\end{array}$ & $\begin{array}{l}6.11 \pm \\
0.00^{a}\end{array}$ \\
\hline Cosford & $\begin{array}{l}35.33 \pm \\
0.46^{\mathrm{bc}}\end{array}$ & $\begin{array}{c}21.54 \pm \\
0.45^{\mathrm{b}}\end{array}$ & $\begin{array}{l}3.88 \pm \\
0.01^{b}\end{array}$ & $\begin{array}{l}4.17 \pm \\
0.10^{\mathrm{bc}}\end{array}$ & $\begin{array}{c}10.77 \pm \\
0.07^{\mathrm{a}}\end{array}$ & $\begin{array}{l}2.72 \pm \\
0.01^{\mathrm{a}}\end{array}$ & $\begin{array}{c}13.79 \pm \\
0.08^{\mathrm{b}}\end{array}$ & $\begin{array}{l}0.26 \pm \\
0.05^{\mathrm{b}}\end{array}$ & $\begin{array}{l}1.55 \pm \\
0.02^{\mathrm{a}}\end{array}$ & $\begin{array}{l}0.68 \pm \\
0.08^{\mathrm{ab}}\end{array}$ & $\begin{array}{l}2.50 \pm \\
0.02^{\mathrm{b}}\end{array}$ & $\begin{array}{l}2.68 \pm \\
0.04^{\mathrm{a}}\end{array}$ & $\begin{array}{l}6.11 \pm \\
0.00^{\mathrm{a}}\end{array}$ \\
\hline Webba Cenny & $\begin{array}{l}34.71 \pm \\
0.43^{c}\end{array}$ & $\begin{array}{l}21.59 \pm \\
0.32^{\mathrm{b}}\end{array}$ & $\begin{array}{l}4.90 \pm \\
0.02^{\mathrm{a}}\end{array}$ & $\begin{array}{l}4.28 \pm \\
0.07^{\mathrm{b}}\end{array}$ & $\begin{array}{c}10.30 \pm \\
0.03^{\mathrm{a}}\end{array}$ & $\begin{array}{l}2.03 \pm \\
0.02 \mathrm{bc}\end{array}$ & $\begin{array}{c}12.95 \pm \\
0.09^{\mathrm{c}}\end{array}$ & $\begin{array}{l}0.27 \pm \\
0.03^{b}\end{array}$ & $\begin{array}{l}1.61 \pm \\
0.02^{a}\end{array}$ & $\begin{array}{l}0.71 \pm \\
0.09 \mathrm{ab}\end{array}$ & $\begin{array}{l}2.50 \pm \\
0.04^{\mathrm{a}}\end{array}$ & $\begin{array}{l}1.76 \pm \\
0.00^{\mathrm{b}}\end{array}$ & $\begin{array}{l}6.09 \pm \\
0.00^{\mathrm{a}}\end{array}$ \\
\hline $\begin{array}{l}\text { Olbrzym z } \\
\text { Halle }\end{array}$ & $\begin{array}{c}39.08 \pm \\
0.15^{\mathrm{a}}\end{array}$ & $\begin{array}{c}25.47 \pm \\
0.11^{\mathrm{a}}\end{array}$ & $\begin{array}{l}3.67 \pm \\
0.15^{\mathrm{bc}}\end{array}$ & $\begin{array}{l}9.13 \pm \\
0.08^{a}\end{array}$ & $\begin{array}{c}10.44 \pm \\
0.20^{\mathrm{a}}\end{array}$ & $\begin{array}{l}2.74 \pm \\
0.00^{\mathrm{a}}\end{array}$ & $\begin{array}{c}13.61 \pm \\
0.06^{\mathrm{b}}\end{array}$ & $\begin{array}{l}0.29 \pm \\
0.02^{b}\end{array}$ & $\begin{array}{l}1.55 \pm \\
0.00^{\mathrm{a}}\end{array}$ & $\begin{array}{l}0.45 \pm \\
0.06^{\mathrm{d}}\end{array}$ & $\begin{array}{l}2.49 \pm \\
0.08^{c}\end{array}$ & $\begin{array}{l}2.72 \pm \\
0.01^{\mathrm{a}}\end{array}$ & $\begin{array}{l}6.11 \pm \\
0.01^{\mathrm{a}}\end{array}$ \\
\hline Kataloński & $\begin{array}{c}32.53 \pm \\
0.50 \mathrm{~d}\end{array}$ & $\begin{array}{l}19.90 \pm \\
0.58^{\mathrm{cd}}\end{array}$ & $\begin{array}{l}3.39 \pm \\
0.08^{\mathrm{c}}\end{array}$ & $\begin{array}{l}3.93 \pm \\
0.01^{\mathrm{c}}\end{array}$ & $\begin{array}{c}10.47 \pm \\
0.12^{\mathrm{a}}\end{array}$ & $\begin{array}{l}2.13 \pm \\
0.08^{\mathrm{b}}\end{array}$ & $\begin{array}{c}12.63 \pm \\
0.07^{\mathrm{c}}\end{array}$ & $0.40 \pm$ & $\begin{array}{l}1.56 \pm \\
0.02^{a}\end{array}$ & $\begin{array}{l}0.55 \pm \\
0.07^{\mathrm{cd}}\end{array}$ & $\begin{array}{l}2.49^{ \pm} \\
0.04^{\mathrm{c}}\end{array}$ & $\begin{array}{l}1.57 \pm \\
0.06^{\mathrm{bc}}\end{array}$ & $\begin{array}{l}6.04 \pm \\
0.00^{a}\end{array}$ \\
\hline Notthingamski & $\begin{array}{l}32.15 \pm \\
0.26^{\mathrm{d}}\end{array}$ & $\begin{array}{c}19.58 \pm \\
0.15^{\mathrm{d}}\end{array}$ & $\begin{array}{l}3.50 \pm \\
0.03 \mathrm{bc}\end{array}$ & $\begin{array}{l}3.95 \pm \\
0.01^{c}\end{array}$ & $\begin{array}{c}10.22 \pm \\
0.08^{\mathrm{a}}\end{array}$ & $\begin{array}{l}1.91 \pm \\
0.07^{\mathrm{c}}\end{array}$ & $\begin{array}{c}12.57^{ \pm} \\
0.04^{\mathrm{c}}\end{array}$ & $\begin{array}{l}0.38^{ \pm} \\
0.08^{b}\end{array}$ & $\begin{array}{l}1.65 \pm \\
0.07^{\mathrm{a}}\end{array}$ & $\begin{array}{l}0.59 \pm \\
0.04^{\mathrm{bc}}\end{array}$ & $\begin{array}{l}2.51 \pm \\
0.01^{\mathrm{a}}\end{array}$ & $\begin{array}{l}1.48^{ \pm} \\
0.09^{\mathrm{c}}\end{array}$ & $\begin{array}{l}6.04 \pm \\
0.00^{\mathrm{a}}\end{array}$ \\
\hline \multicolumn{14}{|l|}{2017} \\
\hline Barceloński & $\begin{array}{l}39.85 \pm \\
0.69 \mathrm{bc}\end{array}$ & $\begin{array}{l}22.90 \pm \\
0.61 \mathrm{abc}\end{array}$ & $\begin{array}{l}5.22 \pm \\
0.16^{\mathrm{a}}\end{array}$ & $\begin{array}{l}5.06 \pm \\
0.07^{\mathrm{a}}\end{array}$ & $\begin{array}{l}11.09 \pm \\
0.12^{\mathrm{ab}}\end{array}$ & $\begin{array}{l}2.47 \pm \\
0.06^{\mathrm{a}}\end{array}$ & $\begin{array}{c}14.19 \pm \\
0.05^{\mathrm{b}}\end{array}$ & $\begin{array}{l}0.30 \pm \\
0.12^{\mathrm{c}}\end{array}$ & $\begin{array}{l}1.56 \pm \\
0.06^{\mathrm{b}}\end{array}$ & $\begin{array}{l}1.03 \pm \\
0.05^{a}\end{array}$ & $\begin{array}{l}2.75 \pm \\
0.02^{c}\end{array}$ & $\begin{array}{l}2.45 \pm \\
0.07^{\mathrm{a}}\end{array}$ & $\begin{array}{l}6.08 \pm \\
0.00^{a}\end{array}$ \\
\hline Cosford & $\begin{array}{c}39.23 \pm \\
1.37^{\mathrm{a}}\end{array}$ & $\begin{array}{l}23.86 \pm \\
1.29 \mathrm{ab}\end{array}$ & $\begin{array}{l}4.71 \pm \\
0.33^{b}\end{array}$ & $\begin{array}{l}5.13 \pm \\
0.19^{a}\end{array}$ & $\begin{array}{c}12.79 \pm \\
0.23^{\mathrm{a}}\end{array}$ & $\begin{array}{l}2.11 \pm \\
0.14^{b}\end{array}$ & $\begin{array}{c}18.89 \pm \\
0.09^{\mathrm{a}}\end{array}$ & $\begin{array}{l}4.87 \pm \\
0.23^{a}\end{array}$ & $\begin{array}{l}1.56 \pm \\
0.14^{\mathrm{b}}\end{array}$ & $\begin{array}{l}1.00 \pm \\
0.09^{\mathrm{a}}\end{array}$ & $\begin{array}{l}2.84 \pm \\
0.08^{\mathrm{bc}}\end{array}$ & $\begin{array}{l}2.52 \pm \\
0.03^{a}\end{array}$ & $\begin{array}{l}6.09 \pm \\
0.00^{\mathrm{a}}\end{array}$ \\
\hline Webba Cenny & $\begin{array}{l}37.95^{ \pm} \\
1.66^{\mathrm{b}}\end{array}$ & $\begin{array}{c}24.22 \pm \\
1.68^{\mathrm{a}}\end{array}$ & $\begin{array}{l}5.24 \pm \\
0.05^{\mathrm{a}}\end{array}$ & $\begin{array}{l}5.30 \pm \\
0.02^{a}\end{array}$ & $\begin{array}{c}12.59 \pm \\
0.12^{\mathrm{a}}\end{array}$ & $\begin{array}{l}1.96 \pm \\
0.03^{b}\end{array}$ & $\begin{array}{l}13.72 \pm \\
0.09 \mathrm{bc}\end{array}$ & $\begin{array}{l}0.38 \pm \\
0.12^{\mathrm{c}}\end{array}$ & $\begin{array}{l}1.56 \pm \\
0.03^{b}\end{array}$ & $\begin{array}{l}1.13 \pm \\
0.09^{a}\end{array}$ & $\begin{array}{l}2.85 \pm \\
0.09 \mathrm{bc}\end{array}$ & $\begin{array}{l}1.71 \pm \\
0.03^{b}\end{array}$ & $\begin{array}{l}6.09 \pm \\
0.00^{a}\end{array}$ \\
\hline $\begin{array}{l}\text { Olbrzym z } \\
\text { Halle }\end{array}$ & $\begin{array}{l}34.11 \pm \\
0.33^{\mathrm{d}}\end{array}$ & $\begin{array}{r}19.99^{ \pm} \\
0.15^{\mathrm{c}}\end{array}$ & $\begin{array}{l}4.53 \pm \\
0.02^{b}\end{array}$ & $\begin{array}{l}4.32 \pm \\
0.11^{b}\end{array}$ & $\begin{array}{l}9.88^{ \pm} \\
0.06^{\mathrm{b}}\end{array}$ & $\begin{array}{l}1.95 \pm \\
0.01^{b}\end{array}$ & $\begin{array}{l}13.82 \pm \\
0.02^{\mathrm{bc}}\end{array}$ & $\begin{array}{l}1.01 \pm \\
0.06^{\mathrm{b}}\end{array}$ & $\begin{array}{l}1.66 \pm \\
0.01^{\mathrm{a}}\end{array}$ & $\begin{array}{l}0.75 \pm \\
0.02^{b}\end{array}$ & $\begin{array}{l}2.85 \pm \\
0.02 \mathrm{bc}\end{array}$ & $\begin{array}{c}1.43 \pm \\
0.08\end{array}$ & $\begin{array}{l}6.11 \pm \\
0.00^{\mathrm{a}}\end{array}$ \\
\hline Kataloński & $\begin{array}{l}34.00 \pm \\
0.17 \mathrm{~cd}\end{array}$ & $\begin{array}{l}21.11 \pm \\
0.10^{\mathrm{bc}}\end{array}$ & $\begin{array}{l}4.71 \pm \\
0.03^{b}\end{array}$ & $\begin{array}{l}5.02 \pm \\
0.08^{a}\end{array}$ & $\begin{array}{l}9.89 \pm \\
0.19^{b}\end{array}$ & $\begin{array}{l}2.00 \pm \\
0.01 \mathrm{~b}\end{array}$ & $\begin{array}{l}13.61 \pm \\
0.01 \mathrm{bc}\end{array}$ & $\begin{array}{l}0.38 \pm \\
0.19^{c}\end{array}$ & $\begin{array}{l}1.63 \pm \\
0.01^{\mathrm{a}}\end{array}$ & $\begin{array}{l}1.00 \pm \\
0.01^{\mathrm{a}}\end{array}$ & $\begin{array}{l}2.95 \pm \\
0.09 \mathrm{ab}\end{array}$ & $\begin{array}{c}1.57 \pm \\
0.03\end{array}$ & $\begin{array}{l}6.07 \pm \\
0.01^{\mathrm{a}}\end{array}$ \\
\hline Notthingamski & $\begin{array}{c}33.93 \pm \\
0.53^{\mathrm{d}}\end{array}$ & $\begin{array}{c}19.93 \pm \\
0.27^{\mathrm{c}}\end{array}$ & $\begin{array}{l}4.27 \pm \\
0.16^{\mathrm{c}}\end{array}$ & $\begin{array}{l}4.20 \pm \\
0.07^{b}\end{array}$ & $\begin{array}{l}9.90 \pm \\
0.25^{\mathrm{b}}\end{array}$ & $\begin{array}{l}1.92 \pm \\
0.05^{b}\end{array}$ & $\begin{array}{c}13.46 \pm \\
0.04^{\mathrm{c}}\end{array}$ & $\begin{array}{l}0.44^{ \pm} \\
0.25^{c}\end{array}$ & $\begin{array}{l}1.63 \pm \\
0.05^{\mathrm{a}}\end{array}$ & $\begin{array}{l}0.68 \pm \\
0.04^{b}\end{array}$ & $\begin{array}{l}3.01 \pm \\
0.01^{\mathrm{a}}\end{array}$ & $\begin{array}{c}1.61 \pm \\
0.04\end{array}$ & $\begin{array}{l}6.09 \pm \\
0.02^{\mathrm{a}}\end{array}$ \\
\hline
\end{tabular}


Table 2. Cont

\begin{tabular}{|c|c|c|c|c|c|c|c|c|c|c|c|c|c|}
\hline & $\begin{array}{c}\text { Total } \\
\text { Polyphenols }\end{array}$ & $\begin{array}{c}\text { Total } \\
\text { Phenolic } \\
\text { Acid }\end{array}$ & $\begin{array}{l}\text { Gallic } \\
\text { Acid }\end{array}$ & $\begin{array}{l}\text { Caffeic } \\
\text { Acid }\end{array}$ & $\begin{array}{c}p \text {-Coumarc } \\
\text { Acid }\end{array}$ & $\begin{array}{l}\text { Ferulic } \\
\text { Acid }\end{array}$ & $\begin{array}{c}\text { Total } \\
\text { Flavonoids }\end{array}$ & $\begin{array}{c}\text { Quercetin- } \\
\text { 3-O- } \\
\text { Rutinoside }\end{array}$ & Catechin & $\begin{array}{l}\text { Epigallo- } \\
\text { Catechin }\end{array}$ & $\begin{array}{c}\text { Kaempferol- } \\
\text { 3-O- } \\
\text { Glucoside }\end{array}$ & Quercetin & Apigenin \\
\hline \multicolumn{14}{|l|}{2018} \\
\hline Barceloński & $\begin{array}{c}36.37 \pm \\
0.36^{\mathrm{c}}\end{array}$ & $\begin{array}{c}22.15 \pm \\
0.21^{\mathrm{c}}\end{array}$ & $\begin{array}{l}4.35 \pm \\
0.10^{\mathrm{b}}\end{array}$ & $\begin{array}{l}4.10 \pm \\
0.06^{\mathrm{d}}\end{array}$ & $\begin{array}{l}11.82 \pm \\
0.03^{\mathrm{b}}\end{array}$ & $\begin{array}{l}1.90 \pm \\
0.15^{\mathrm{b}}\end{array}$ & $\begin{array}{c}14.36 \pm \\
0.03^{\mathrm{b}}\end{array}$ & $\begin{array}{l}0.30 \pm \\
0.03^{a b}\end{array}$ & $\begin{array}{l}1.55 \pm \\
0.02^{\mathrm{c}}\end{array}$ & $\begin{array}{l}1.45 \pm \\
0.02^{\mathrm{a}}\end{array}$ & $\begin{array}{l}3.25 \pm \\
0.01^{\mathrm{a}}\end{array}$ & $\begin{array}{l}1.61 \pm \\
0.02^{c}\end{array}$ & $\begin{array}{l}6.08 \pm \\
0.01^{\mathrm{a}}\end{array}$ \\
\hline Cosford & $\begin{array}{c}33.62 \pm \\
0.29^{\mathrm{e}}\end{array}$ & $\begin{array}{c}20.29 \pm \\
0.311^{\mathrm{e}}\end{array}$ & $\begin{array}{l}4.38 \pm \\
0.02^{b}\end{array}$ & $\begin{array}{l}4.25 \pm \\
0.01^{\mathrm{cd}}\end{array}$ & $\begin{array}{l}9.73 \pm \\
0.00^{c}\end{array}$ & $\begin{array}{l}2.07 \pm \\
0.03 \mathrm{ab}\end{array}$ & $\begin{array}{c}13.48^{ \pm} \\
0.05^{\mathrm{c}}\end{array}$ & $\begin{array}{c}0.48^{ \pm} \\
0.00^{\mathrm{a}}\end{array}$ & $\begin{array}{l}1.59 \pm \\
0.03^{b}\end{array}$ & $\begin{array}{l}0.84 \pm \\
0.05^{\mathrm{b}}\end{array}$ & $\begin{array}{l}3.04 \pm \\
0.01^{b}\end{array}$ & $\begin{array}{l}1.50 \pm \\
0.05^{\mathrm{cd}}\end{array}$ & $\begin{array}{c}6.03 \pm \\
0.01^{\mathrm{a}}\end{array}$ \\
\hline Webba Cenny & $\begin{array}{c}43.30 \pm \\
0.23^{\mathrm{a}}\end{array}$ & $\begin{array}{c}29.26 \pm \\
0.31^{\mathrm{a}}\end{array}$ & $\begin{array}{l}3.96 \pm \\
0.05^{\mathrm{c}}\end{array}$ & $\begin{array}{l}3.89 \pm \\
0.14^{\mathrm{e}}\end{array}$ & $\begin{array}{c}19.62 \pm \\
0.17^{\mathrm{a}}\end{array}$ & $\begin{array}{l}2.07 \pm \\
0.04 \mathrm{ab}\end{array}$ & $\begin{array}{c}14.44^{ \pm} \\
0.06^{\mathrm{b}}\end{array}$ & $\begin{array}{l}0.49 \pm \\
0.17^{\mathrm{a}}\end{array}$ & $\begin{array}{l}1.55 \pm \\
0.04^{\mathrm{c}}\end{array}$ & $\begin{array}{l}0.76 \pm \\
0.06^{\mathrm{b}}\end{array}$ & $\begin{array}{l}2.79 \pm \\
0.14^{\mathrm{c}}\end{array}$ & $\begin{array}{l}2.69 \pm \\
0.06^{\mathrm{b}}\end{array}$ & $\begin{array}{l}6.06 \pm \\
0.00^{a}\end{array}$ \\
\hline $\begin{array}{l}\text { Olbrzym z } \\
\text { Halle }\end{array}$ & $\begin{array}{l}36.13 \pm \\
0.14^{\mathrm{cd}}\end{array}$ & $\begin{array}{l}21.11 \pm \\
0.17^{\mathrm{d}}\end{array}$ & $\begin{array}{l}4.52 \pm \\
0.02 \mathrm{~b}\end{array}$ & $\begin{array}{l}4.59 \pm \\
0.03^{a}\end{array}$ & $\begin{array}{c}10.12 \pm \\
0.04^{\mathrm{c}}\end{array}$ & $\begin{array}{l}2.02 \pm \\
0.01 \mathrm{ab}\end{array}$ & $\begin{array}{c}15.02 \pm \\
0.09^{\mathrm{a}}\end{array}$ & $\begin{array}{l}0.21 \pm \\
0.04^{b}\end{array}$ & $\begin{array}{l}1.57 \pm \\
0.01 \mathrm{bc}\end{array}$ & $\begin{array}{l}0.87 \pm \\
0.09^{b}\end{array}$ & $\begin{array}{l}2.84 \pm \\
0.08^{c}\end{array}$ & $\begin{array}{l}3.50 \pm \\
0.09^{\mathrm{a}}\end{array}$ & $\begin{array}{l}6.03 \pm \\
0.00^{a}\end{array}$ \\
\hline Kataloński & $\begin{array}{c}37.03 \pm \\
0.34^{\mathrm{b}}\end{array}$ & $\begin{array}{c}24.75 \pm \\
0.12^{\mathrm{b}}\end{array}$ & $\begin{array}{l}8.43 \pm \\
0.04^{\mathrm{a}}\end{array}$ & $\begin{array}{l}4.38 \pm \\
0.05^{\mathrm{bc}}\end{array}$ & $\begin{array}{c}10.10 \pm \\
0.06^{\mathrm{c}}\end{array}$ & $\begin{array}{l}1.87 \pm \\
0.02^{b}\end{array}$ & $\begin{array}{c}12.88 \pm \\
0.00^{\mathrm{d}}\end{array}$ & $\begin{array}{l}0.30 \pm \\
0.06^{\mathrm{b}}\end{array}$ & $\begin{array}{l}1.56 \pm \\
0.02 \mathrm{bc}\end{array}$ & $\begin{array}{l}0.75 \pm \\
0.00^{\mathrm{b}}\end{array}$ & $\begin{array}{l}2.76 \pm \\
0.02^{c}\end{array}$ & $\begin{array}{l}1.44 \pm \\
0.00^{\mathrm{d}}\end{array}$ & $\begin{array}{l}6.07 \pm \\
0.07^{\mathrm{a}}\end{array}$ \\
\hline Notthingamski & $\begin{array}{l}35.60 \pm \\
0.11^{\mathrm{d}}\end{array}$ & $\begin{array}{l}22.18 \pm \\
0.16^{\mathrm{c}}\end{array}$ & $\begin{array}{c}3.812^{ \pm} \\
0.03^{c}\end{array}$ & $\begin{array}{l}4.55 \pm \\
0.03 \mathrm{ab}\end{array}$ & $\begin{array}{c}12.06 \pm \\
0.03^{\mathrm{b}}\end{array}$ & $\begin{array}{l}2.20 \pm \\
0.01^{\mathrm{a}}\end{array}$ & $\begin{array}{r}13.33^{ \pm} \\
0.03^{c}\end{array}$ & $\begin{array}{l}0.44 \pm \\
0.03^{\mathrm{ab}}\end{array}$ & $\begin{array}{l}1.68 \pm \\
0.01^{\mathrm{a}}\end{array}$ & $\begin{array}{l}0.81 \pm \\
0.03^{b}\end{array}$ & $\begin{array}{l}2.80 \pm \\
0.01^{\mathrm{c}}\end{array}$ & $\begin{array}{l}1.51 \pm \\
0.07^{\mathrm{cd}}\end{array}$ & $\begin{array}{l}6.08 \pm \\
0.07^{\mathrm{a}}\end{array}$ \\
\hline $\begin{array}{l}p \text {-Value } \\
\text { cultivar } 2016\end{array}$ & $<0.0001$ & $<0.0001$ & $<0.0001$ & $<0.0001$ & N.S & $<0.0001$ & $<0.0001$ & $<0.0001$ & N.S & $<0.0001$ & 0.0028 & $<0.0001$ & N.S \\
\hline $\begin{array}{l}p \text {-Value } \\
\text { cultivar } 2017\end{array}$ & $<0.0001$ & 0.001 & $<0.0001$ & $<0.0001$ & 0.005 & 0.002 & $<0.0001$ & $<0.0001$ & 0.003 & $<0.0001$ & $<0.0001$ & $<0.0001$ & N.S \\
\hline $\begin{array}{l}p \text {-Value } \\
\text { cultivar } 2018\end{array}$ & $<0.0001$ & $<0.0001$ & $<0.0001$ & 0.0132 & $<0.0001$ & 0.003 & $<0.0001$ & 0.016 & $<0.0001$ & $<0.0001$ & $<0.0001$ & $<0.0001$ & N.S \\
\hline$p$-Value year & N.S ** & N.S & N.S & N.S & N.S & $<0.0001$ & 0.043 & 0.029 & 0.042 & $<0.0001$ & $<0.0001$ & N.S & N.S \\
\hline $\begin{array}{l}\text { Interactions } \\
\text { year* cultivar }\end{array}$ & $<0.0001$ & $<0.0001$ & $<0.0001$ & N.S & 0.004 & $<0.0001$ & $<0.0001$ & 0.003 & N.S & 0.0052 & N.S & $<0.0001$ & N.S \\
\hline
\end{tabular}

${ }^{*}$ Values followed by different small letters (a-e) in the same column are significantly different $(p<0.05) .{ }^{* *}$ N.S-not significant statistically. 


\subsubsection{Tocopherol Content}

The concentrations of $\beta+y$-tocopherol, $\delta$-tocopherol and $\alpha$-tocopherol and total tocopherols found in the six hazelnut cultivars are given in Table 3. $\alpha$-Tocopherol was the most abundant tocopherol, followed by $\beta+Y$-tocopherol and $\delta$-tocopherol in all investigated raw and roasted hazelnut cultivars. The statistical analysis showed that roasting and the year of cultivation did not affect the composition of the investigated samples. However, differences between cultivars were observed among all six cultivars, beside $\delta$-tocopherol in raw hazelnut kernels. $\alpha$-Tocopherol ranged from 25.77 to $31.90 \mathrm{mg}$

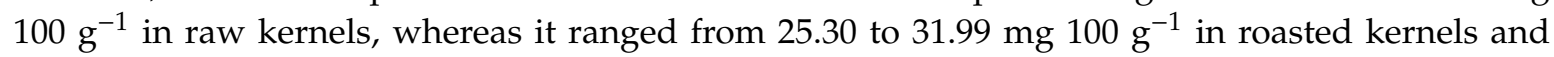
represented approximately $86-92 \%$ of the total tocopherols. The highest percent concentration of $\alpha$-tocopherol (91\%) was observed in the 'Kataloński' and 'Olbrzym z Halle' cultivars; accordingly the lowest concentrations of $\beta+y$-tocopherol and $\delta$-tocopherol were 2.41 and 0.22 , and 2.27 and $0.31 \mathrm{mg}$ $100 \mathrm{~g}^{-1}$, respectively. The lowest concentration of $\alpha$-tocopherol was observed in the 'Webba Cenny' cultivar, and consequently, the concentrations of $\beta+y$-tocopherol and $\delta$-tocopherol were the highest

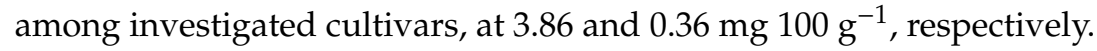


Table 3. Tocopherols content (mg $100 \mathrm{~g}^{-1}$ ) in oil obtained from six raw and roasted hazelnuts cultivars in 2016-2018 (mean \pm SD).

\begin{tabular}{|c|c|c|c|c|c|c|c|c|c|}
\hline \multicolumn{5}{|c|}{ RAW } & \multicolumn{5}{|c|}{ ROASTED } \\
\hline & $\begin{array}{c}\beta+ \\
y \text {-Tocopherol }\end{array}$ & $\delta$-Tocopherol & $\alpha$-Tocopherol & Total & $\begin{array}{c}\beta+ \\
y \text {-Tocopherol }\end{array}$ & $\delta$-Tocopherol & $\alpha$-Tocopherol & Total & $\begin{array}{l}p \text {-Value } \\
\text { Roasting }\end{array}$ \\
\hline Barceloński & $2.68 \pm 0.38^{b^{*}}$ & $0.27 \pm 0.05^{a}$ & $28.67 \pm 3.99 \mathrm{ab}$ & $31.62 \pm 4.10^{\mathrm{ab}}$ & $2.70 \pm 0.23^{a b}$ & $0.27 \pm 0.05^{\mathrm{ab}}$ & $28.60 \pm 3.41^{b}$ & $31.57 \pm 2.32^{b}$ & N.S \\
\hline Cosford & $3.17 \pm 0.43^{a}$ & $0.27 \pm 0.05^{a}$ & $25.77 \pm 3.60^{c}$ & $29.21 \pm 3.71^{c}$ & $3.16 \pm 0.21^{a}$ & $0.26 \pm 0.06^{b}$ & $25.41 \pm 2.40^{c}$ & $28.83 \pm 2.81^{c}$ & N.S \\
\hline Kataloński & $2.41 \pm 0.33^{b}$ & $0.22 \pm 0.05^{\mathrm{a}}$ & $29.60 \pm 4.16^{\mathrm{a}}$ & $32.23 \pm 4.21 \mathrm{ab}$ & $2.40 \pm 0.21^{b}$ & $0.22 \pm 0.05^{b}$ & $29.01 \pm 4.12 \mathrm{ab}$ & $31.63 \pm 1.56^{b}$ & N.S \\
\hline Nottinghamski & $3.10 \pm 0.44^{a}$ & $0.32 \pm 0.06^{\mathrm{a}}$ & $31.90 \pm 4.49^{a}$ & $35.32 \pm 4.55^{\mathrm{a}}$ & $3.11 \pm 0.44^{\mathrm{a}}$ & $0.29 \pm 0.06^{\mathrm{a}}$ & $31.99 \pm 3.52^{\mathrm{a}}$ & $35.39 \pm 3.56^{\mathrm{a}}$ & N.S \\
\hline Olbrzym z Halle & $2.27 \pm 0.31^{b}$ & $0.31 \pm 0.06^{\mathrm{a}}$ & $26.90 \pm 3.78^{b}$ & $29.48 \pm 3.84^{c}$ & $2.23 \pm 0.11^{b}$ & $0.31 \pm 0.04^{\mathrm{a}}$ & $25.92 \pm 3.32^{c}$ & $28.46 \pm 4.14^{\mathrm{d}}$ & N.S \\
\hline Webba Cenny & $3.86 \pm 0.53^{a}$ & $0.36 \pm 0.06^{\mathrm{a}}$ & $26.41 \pm 3.68^{b}$ & $30.63 \pm 3.80^{b}$ & $3.82 \pm 0.33^{a}$ & $0.37 \pm 0.05^{\mathrm{a}}$ & $25.40 \pm 3.60^{c}$ & $29.59 \pm 2.82^{d}$ & N.S \\
\hline$p$-value cultivar & 0.039 & N.S ** & $<0.0001$ & $<0.0001$ & 0.045 & 0.001 & $<0.0001$ & $<0.0001$ & \\
\hline
\end{tabular}

*Values followed by different small letters $(\mathrm{a}-\mathrm{d})$ in the same column are significantly different $(p<0.05) .{ }^{* *}$ N.S-not significant statistically. 


\subsection{Principal Component Analysis}

A principal component analysis (PCA) was performed to summarize the variations between the hazelnut cultivars through the roasting process over the 3 seasons of experiment. Score plot $\mathrm{A}$ (Figure 2A) showed that the two first components described $98.82 \%$ (percentage of variance $94.54 \%$ and $4.28 \%$, respectively) of the total variance. The PCA allows for the discrimination of hazelnut into two groups situated in different areas of the plot. The first group of raw hazelnut with skin and roasted hazelnuts kernels without skin had a negative value along the horizontal axis and presented only a small amount of diversity of variation from the tested samples, regardless of the year of the study. Raw hazelnuts kernels were not separated from their corresponding roasted samples without skin. The hazelnut skin S16 and S17 were positive along vertical and horizontal axes, whereas S18 showed as negative along the vertical axes and positive along the horizontal axes, with the differences between skin samples being due to the different content of flavonoids, which were also previously described. The PCA score plot confirmed that the roasting process led to changes in the content of phenolic compounds in hazelnut skin.

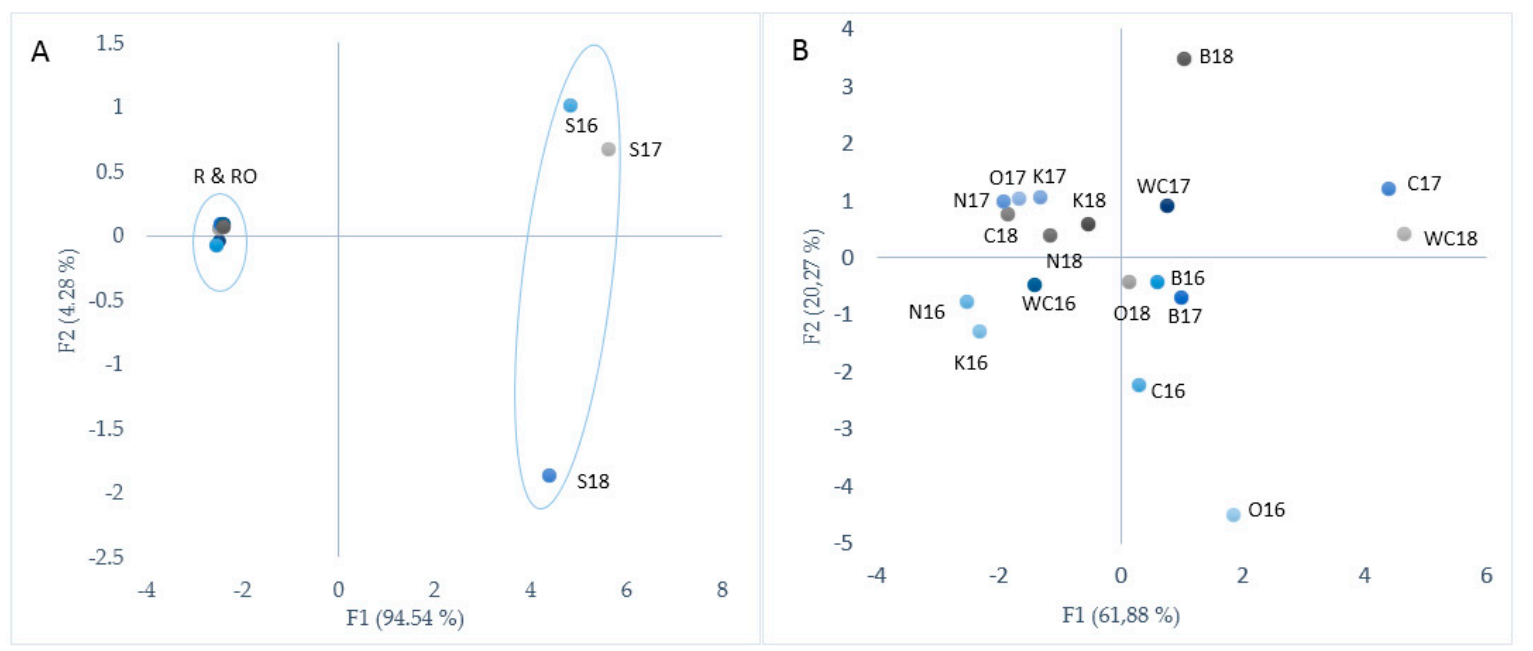

Figure 2. Principal components analysis score plots of raw and roasted hazelnut kernels and its skin (A), and among six hazelnut cultivars (B) over three years of experiment. (B-Barceloński, K-Katalonski, WC-Webba Cenny, C-Cosford, O-Olbrzym z Halle, N-Notthingamski, R-raw hazelnut, RO—roasted hazelnut, S—skin, 16-2016, 17-2017, 18-2018).

The first two components ( $\mathrm{F} 1$ and $\mathrm{F} 2,61.88 \%$ and $20.27 \%$. respectively) explained $82.15 \%$ of the total variation (Figure 2B), showing unclear discrimination between hazelnut cultivars, as well as year of experiment. The visualization of the third principal component (F3: $18.22 \%$ of the total variance) was not useful in improving the samples separation. However, is possible to note areas were some small clusters are observable. The hazelnut cultivars from 2016 were negative along vertical axis, and create two groups from tested samples N16, K16, WC16 and O16, C16, B16. The cultivars 'Kataloński' and 'Notthingamski' from 2017 and 2018 were grouped in the negative part of the horizontal axes and were positive along the vertical axes, highlighting similarities between cultivars and year of experiment. The C18 and $\mathrm{O} 17$ were clearly separated from their corresponding samples (C17, C16, O16, O18).

\section{Discussion}

The contents of bioactive compounds in hazelnuts depend on many factors such as agricultural practices, and the cultivar and their characteristics can vary considerably [25]. Factors such as altitude, geographical area, and temperature during the growing period showed a strong relationship with the total phenol content in the investigated hazelnut cultivars. Flavonoids are carbon-based products of the secondary metabolism accumulated in the outer and aerial tissues, and act as powerful photoprotectants 
and antioxidants. Exposure to light has a considerable effect on most flavonoids [26,27], which is consistent with the results obtained in our study, where the highest flavonoid content was observed in 2017, when the greatest amount of sunlight per month occurred. Phenolic acids are directly involved in the response of plants to different types of stress they contribute to healing via the lignification of damaged areas, and possess antimicrobial properties, with their concentrations potentially increasing after infection. Furthermore, plants grown in cold climates often present stronger antioxidant properties, as this one of strategies for countering oxidative stress [28].

The results of the HPLC identification of the phenolic composition of hazelnut kernels were in accord with previously reported data from different hazelnut cultivars [21,29-32], except for proto-catechuic acid, which was widely detected in hazelnuts and their byproducts. Additionally, to the best of our knowledge apigenin was detected for the first time in hazelnuts, in which it was found to be the predominant flavonol in both roasted and unroasted kernels. Kumar et al. [33] reported that apigenin was detected in the hard shell of Corylus jacquemontii, as well as in the pistachio hard shell [34]. Apigenin is found in significant quantities in parsley, celery, oranges, thyme, onions, honey, and various spices as well as beverages derived from plants such as tea, beer and wine [35]. Furthermore, the six investigated cultivars showed a similar profiles of phenolic compounds and no single hazelnut cultivar could be regarded as the best source relative to the others.

After the roasting process, the total polyphenol content significantly increases due to the release of bound phenolic compounds and the formation of Maillard reaction products such as melanoidins [11]. As reported by Lee et al. [36] plants contain considerable amounts of bound form antioxidant phenolics and thermal processing might break down these covalently bonded polymeric compounds to liberate them into free forms, which enhances their antioxidant capacity. The decrease in polyphenols after roasting is due to the removal of the brown hazelnut skin (perisperm), which is an important source of phenolic compounds, containing approximately 40-fold higher levels of polyphenols than the kernels [37]. Locatelli et al. [37] and Pelvan et al. [21] observed that the antioxidant activity of hazelnut decreased as a consequence of roasting. Marzocchi et al. [38] demonstrated that the total phenolic compounds tended to increase as a result of roasting in the 'Kataloński' cultivar grown in Poland. Similar results were obtained by Lucchetti et al. [39]. Furthermore, other authors have indicated that thermal processing does not impact the polyphenol content, which is consistent with our results [40]. The polyphenol content can differ depending on the food matrix, roasting conditions, and applied extraction protocols. In the present study, the applied roasting temperature was lower, than that used in other studies. In addition to the roasting conditions, the amounts of compounds involved in the Maillard reaction, particularly carbohydrates and proteins will determine the final polyphenol content in roasted nuts [41]. The results previously obtained by Król et al. [42] for the same hazelnut cultivars, showed that cultivars grown in Poland exhibited higher average carbohydrate contents and lower average protein contents compared to hazelnuts originating from Turkey, Spain or Italy. This could have a positive effect on the balance between the thermal degradation of naturally occurring antioxidant compounds and the formation of new Maillard reaction products enhancing antioxidant activity [43]. The majority of phenolic acids are located in the roasted hazelnut skin which is removed from raw hazelnuts during the roasting process. The presentation of total polyphenols from all investigated cultivars seems to be more appropriate because the separated skin will be used as a byproduct of the whole processing line, not only one cultivar. Hazelnut skin contains more phenolic compounds than some of the most antioxidant-rich foods such as coffee, green tea, dark chocolate and cinnamon [4]. Tas and Gokmen [4], Pelvan et al. [21] and Shahidi et al. [44] also reported that gallic acid was the most abundant phenolic acid in hazelnut skin and that the values varied among cultivars of hazelnut, which is consistent with our results, whereas Del Rio et al. [45] reported that protocatechuic acid was the predominant phenolic acid in hazelnut skin. Additionally, we showed the presence of rutin in hazelnut skin, in contrast to what has been reported in the literature [4].

Tocopherols are good lipid-soluble antioxidants present in all plant oils. They may protect oils from oxidative deterioration and show vitamin E activity in the human body. Vitamin E consists of four 
isoforms $(\beta, y, \delta, \alpha)$ of tocopherol and tocotrienol. The concentrations of $\alpha-, \beta-, \delta$ - and $\gamma$-tocopherols determined in the present study were higher than those found in other hazelnut cultivars $[39,46]$, but much lower than those obtained by Marzocchi et al. [38]. Moreover, Marzocchi et al. [38] showed, that roasting conditions significantly affect the tocopherols contents compared to those found in raw hazelnuts, which differ from the findings of our study, whereas Amaral et al. [9] showed that tocopherol levels in hazelnut kernels are quite stable during roasting, which is in close agreement with our results. Alamprese et al. [47] indicated that tocopherols are resistant to thermal degradation and that the loss of tocopherols is not affected by the temperature of the roasting process [46]. Additionally, the skin could provide protection to nut samples during roasting, preventing the thermal degradation of tocopherols.

\section{Conclusions}

The bioactive profile and the distribution of bioactive compounds in six hazelnut cultivars were measured in this study. Statistical analysis showed that the year of cultivation had a significant effect on the polyphenol content of hazelnut kernels ( $p$-coumaric, ferulic, quercetin-3-O-rutinoside, kaempferol-3-O-glucoside, and epigallocatechin). Among the detected phenolic acids, $p$-coumaric acid was the most abundant in roasted and unroasted kernels, whereas apigenin was the most abundant flavonol. Additionally, to the best of our knowledge, apigenin was detected for the first time in hazelnuts in the present study, where it was found to be the predominant flavonol in whole roasted and unroasted kernels. Roasting had a minor impact on the polyphenol content of roasted hazelnut kernels without skin. The majority of phenolic acids were located in the roasted hazelnut skin, which was found to contain 168- to 378- fold higher contents of total phenolic compounds than raw and roasted nuts. The content of tocopherols during roasting was quite stable, and no significant influence was observed. The six investigated cultivars showed similar profiles of phenolic compounds, and no single hazelnut cultivar could be regarded as the best source relative to the others.

Author Contributions: Conceptualization. K.K.; methodology. K.K. and E.H.; software. K.K. and E.H.; validation. M.G.; formal analysis. K.K.; investigation. K.K. M.G. and E.H.; resources. K.K.; data curation. M.G.; writing-original draft preparation. K.K.; writing—review and editing. K.K.; visualization. K.K.; supervision. M.G. and A.P.; project administration. M.G.; funding acquisition. A.P., E.H. and M.G. All authors have read and agreed to the published version of the manuscript.

Funding: The Polish Federation of Food Industry Union of Employers (PFFI UE): Research Grant of PFFI UE 2019

Acknowledgments: This work was supported by the Institute of Human Nutrition. Warsaw University of Life Sciences (WULS) for scientific research.

Conflicts of Interest: The authors declare no conflict of interest. 


\section{Appendix A}
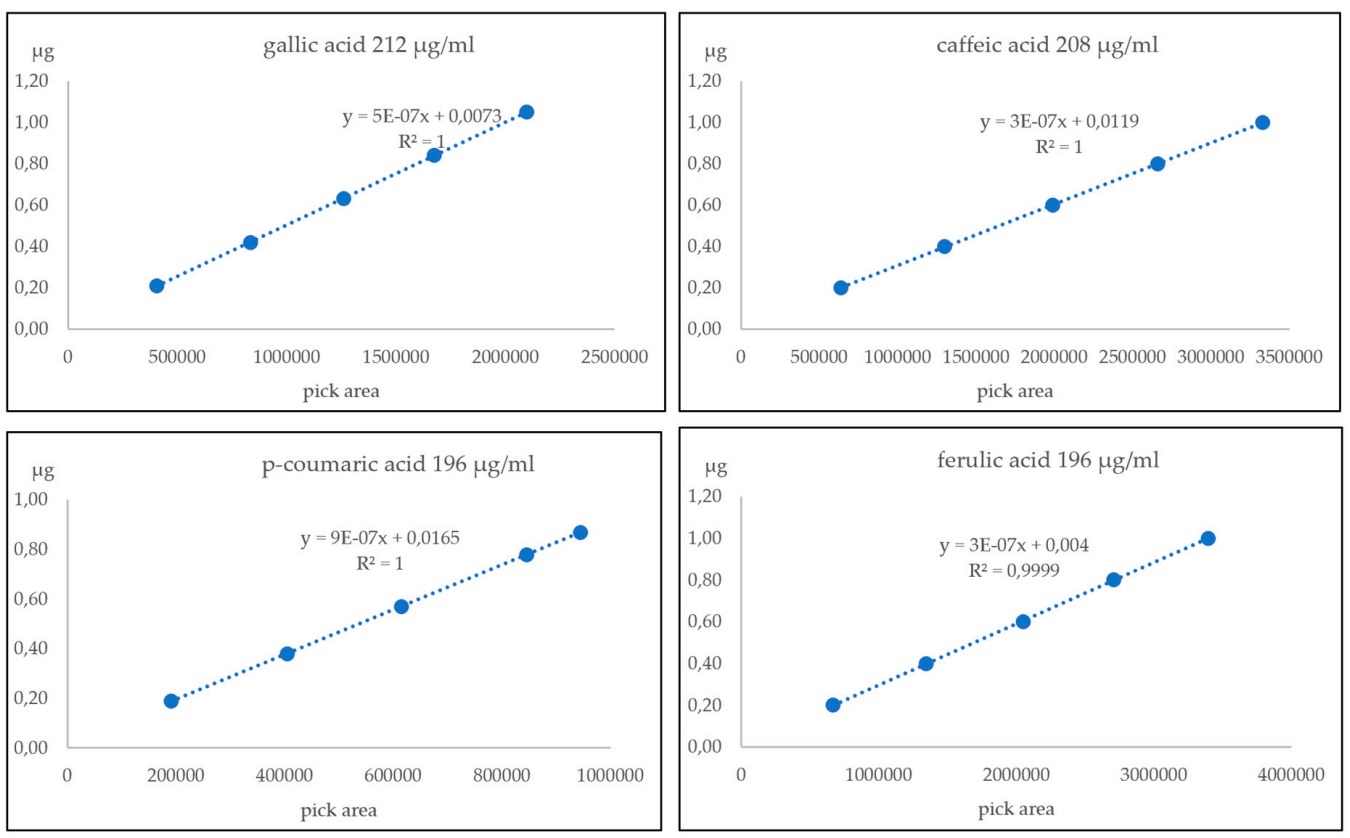

Figure A1. Standard curves used for identification for phenolic acids.
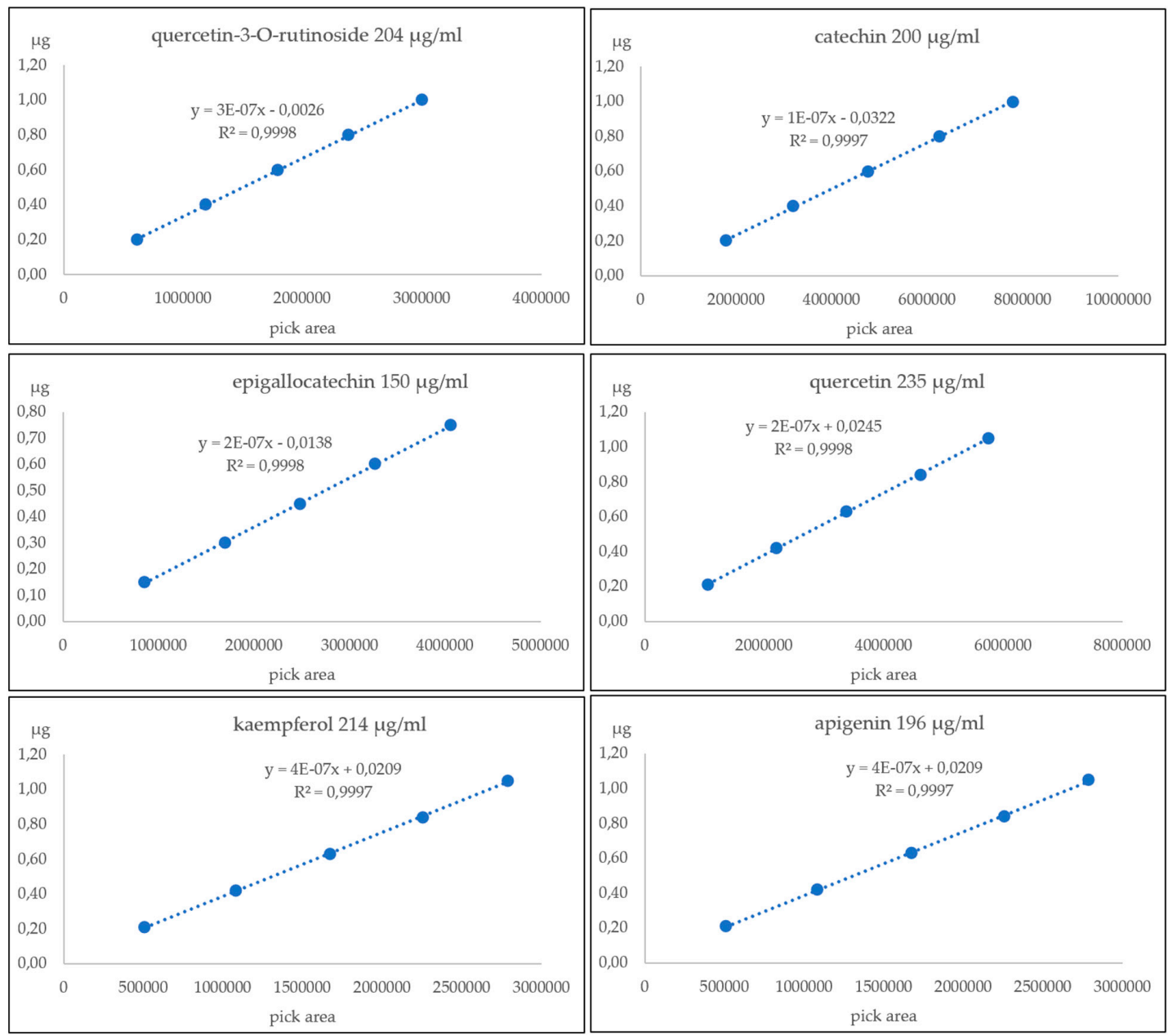

Figure A2. Standard curves used for identification for flavonoids. 


\section{References}

1. FAOSTAT. Food and Agricultural Organization of the United Nations. 2018. Available online: http: //www.fao.org/faostat/en/\#data/QC (accessed on 10 August 2019).

2. Gantner, M. Susceptibility of large-fruited hazel cultivars grown in Poland to major pest and their crop productivity. Acta Hortic. 2005, 686, 377-384. [CrossRef]

3. Chang, S.K.; Alasalvar, C.; Bolling, B.W.; Shahidi, F. Nuts and their co-products: The impact of processing (roasting) on phenolics, bioavailability. and health benefits-A comprehensive review. J. Funct. Foods 2016, 26, 88-122. [CrossRef]

4. Taş, N.G.; Gökmen, V. Phenolic compounds in natural and roasted nuts and their skins: A brief review. Curr. Opin. Food Sci. 2017, 14, 103-109. [CrossRef]

5. Caimari, A.; Puiggròs, F.; Suárez, M.; Crescenti, A.; Laos, S.; Ruiz, J.A.; Alonso, V.; Moragas, J.; del Bas, J.M.; Arola, L. The intake of a hazelnut skin extract improves the plasma lipid profile and reduces the lithocholic/deoxycholic bile acid faecal ratio, a risk factor for colon cancer, in hamsters fed a high-fat diet. Food Chem. 2015, 167, 138-144. [CrossRef]

6. Bolling, B.W.; Chen, C.O.; Mckay, D.L.; Blumberg, J.B. Tree nut phytochemicals: Composition, antioxidant capacity, bioactivity, impact factors. A systematic review of almonds, Brazils, cashews, hazelnuts, macadamias, pecans, pine nuts, pistachios and walnuts. Nutr. Res. Rev. 2011, 24, 244-275. [CrossRef]

7. Kornsteriner, M.; Wagner, K.H.; Elmadfa, I. Tocopherols and total phenolics in 10 different nut types. Food Chem. 2006, 98, 381-387. [CrossRef]

8. Alasalvar, C.; Shahidi, F.; Ohshima, T.; Wanasundara, U.; Yurttas, H.C.; Liyanapathirana, C.M.; Rodrigues, F.B. Turkish Tombul Hazelnut (Corylus avellana L.). 2. Lipid Characteristics and Oxidative Stability. J. Agric. Food Chem. 2003, 51, 3797-3805. [CrossRef]

9. Amaral, J.S.; Casal, S.; Citová, I.; Santos, A.; Seabra, R.M.; Oliveira, B.P.P. Characterization of several hazelnut (Corylus avellana L.) cultivars based in chemical, fatty acid and sterol composition. Eur. Food Res. Technol. 2006, 222, 274-280. [CrossRef]

10. Wang, W.; Jung, J.; McGorrin, R.J.; Traber, M.G.; Leonard, S.W.; Cherian, G.; Zhao, Y. Investigation of drying conditions on bioactive compounds. lipid oxidation. and enzyme activity of Oregon hazelnuts (Corylus avellana L.). LWT 2018, 90, 526-534. [CrossRef]

11. Chandrasekara, N.; Shahidi, F. Effect of Roasting on Phenolic Content and Antioxidant Activities of Whole Cashew Nuts, Kernels, and Testa. J. Agric. Food Chem. 2011, 59, 5006-5014. [CrossRef]

12. Köksal, A.I.; Artik, N.; Şimşek, A.; Güneş, N. Nutrient composition of hazelnut (Corylus avellana L.) varieties cultivated in Turkey. Food Chem. 2006, 99, 509-515. [CrossRef]

13. Donno, D.; Beccaro, G.L.; Mellano, G.M.; Prima, S.D.; Cavicchioli, M.; Cerutti, A.K.; Bounous, G. Setting a protocol for hazelnut roasting using sensory and colorimetric analysis: Influence of the roasting temperature on the quality of Tonda Gentile delle Langhe cv. Hazelnut. Czech J. Food Sci. 2013, 31, 390-400. [CrossRef]

14. Lainas, K.; Alasalvar, C.; Bolling, B.W. Effects of roasting on proanthocyanidin contents of Turkish Tombul hazelnut and its skin. J. Funct. Foods 2016, 23, 647-653. [CrossRef]

15. Montella, R.; Coïsson, J.D.; Travaglia, F.; Locatelli, M.; Malfa, P.; Martelli, A.; Arlorio, M. Bioactive compounds from hazelnut skin (Corylus avellana L.): Effects on Lactobacillus plantarum P17630 and Lactobacillus crispatus P17631. J. Funct. Foods 2013, 5, 306-315. [CrossRef]

16. Zeppa, G.; Belviso, S.; Bertolino, M.; Cavallero, M.C.; Dal Bello, B.; Ghirardello, D.; Giordano, M.; Giorgis, M.; Grosso, A.; Rolle, L.; et al. The effect of hazelnut roasted skin from different cultivars on the quality attributes. polyphenol content and texture of fresh egg pasta. J. Sci. Food Agric. 2015, 95, 1678-1688. [CrossRef]

17. Bertolino, M.; Belviso, S.; Dal Bello, B.; Ghirardello, D.; Giordano, M.; Rolle, L.; Gerbi, V.; Zeppa, G. Influence of the addition of different hazelnut skins on the physicochemical, antioxidant, polyphenol and sensory properties of yogurt. LWT Food Sci. Technol. 2015, 63, 1145-1154. [CrossRef]

18. Özdemir, K.S.; Yılmaz, C.; Durmaz, G.; Gökmen, V. Hazelnut skin powder: A new brown colored functional ingredient. Food Res. Int. 2014, 65, 291-297. [CrossRef]

19. Cikrikci, S.; Demirkesen, I.; Mert, B. Production of hazelnut skin fibres and utilisation in a model bakery product. Qual. Assur. Saf. Crop. Foods 2016, 8, 195-206. [CrossRef]

20. Jakopic, J.; Petkovsek, M.M.; Likozar, A.; Solar, A.; Stampar, F.; Veberic, R. HPLC-MS identification of phenols in hazelnut (Corylus avellana L.) kernels. Food Chem. 2011, 124, 1100-1106. [CrossRef] 
21. Pelvan, E.; Olgun, E.Ö.; Karadağ, A.; Alasalvar, C. Phenolic profiles and antioxidant activity of Turkish Tombul hazelnut samples (natural, roasted, and roasted hazelnut skin). Food Chem. 2018, 244, 102-108. [CrossRef]

22. Meteorological station of Polish Institute of Meteorology and Water Management. Available online: https://danepubliczne.imgw.pl/\#dane-synoptyczne (accessed on 11 November 2019).

23. Polish National List of Fruit Plant Varieties. Available online: http://www.coboru.pl/Polska/Rejestr/odm_w_ rej.aspx?kodgatunku=LEDO (accessed on 28 October 2019).

24. Determination of Tocopherol and Tocotrienol Contents by High-Performance Liquid Chromatography PN-EN ISO 9936:2016-05 (ISO 9936:2016). Available online: https://www.iso.org/standard/69595.html (accessed on 1 August 2019).

25. Coğrafya, M.; Sayi, D. The effect of climatic conditions on hazelnut (Corylus avellana) yield in Giresun (Turkey). Marmara Coğrafya Derg. Sayi. 2012, 26, 302-323.

26. Manach, C.; Scalbert, A.; Morand, C.; Rémésy, C.; Jiménez, L. Polyphenols: Food sources and bioavailability. Am. J. Clin. Nutr. 2004, 79, 727-747. [CrossRef] [PubMed]

27. Agati, G.; Azzarello, E.; Pollastri, S.; Tattini, M. Flavonoids as antioxidants in plants: Location and functional significance. Plant Sci. 2012, 196, 67-76. [CrossRef] [PubMed]

28. Tolić, M.T.; Krbavčić, I.P.; Vujević, P.; Milinović, B.; Jurčević, I.L.; Vahčić, N. Effects of weather conditions on phenolic content and antioxidant capacity in juice of chokeberries (Aronia melanocarpa L.). Pol. J. Food Nutr. Sci. 2017, 67, 67-74. [CrossRef]

29. Kalogeropoulos, N.; Chiou, A.; Ioannou, M.S.; Karathanos, V.T. Nutritional evaluation and health promoting activities of nuts and seeds cultivated in Greece. Int. J. Food Sci. Nutr. 2013, 64, 757-767. [CrossRef]

30. Gültekin-Özgüven, M.; Davarci, F.; Pasli, A.A.; Demir, N.; Özçelik, B. Determination of phenolic compounds by ultra high liquid chromatography-tandem mass spectrometry: Applications in nuts. LWT Food Sci. Technol. 2015, 64, 42-49. [CrossRef]

31. Ciemniewska-Zytkiewicz, H.; Verardo, V.; Pasini, F.; Bryś, J.; Koczoń, P.; Caboni, M.F. Determination of lipid and phenolic fraction in two hazelnut (Corylus avellana L.) cultivars grown in Poland. Food Chem. 2015, 168, 615-622. [CrossRef]

32. Slatnar, A.; Mikulic-Petkovsek, M.; Stampar, F.; Veberic, R.; Solar, A. HPLC-MSn identification and quantification of phenolic compounds in hazelnut kernels, oil and bagasse pellets. Food Res. Int. 2014, 64, 783-789. [CrossRef]

33. Kumar, A.; Kumar, P.; Koundal, R.; Agnihotri, V.K. Antioxidant properties and UPLC-MS/MS profiling of phenolics in jacquemont's hazelnut kernels (Corylus jacquemontii) and its byproducts from western Himalaya. J. Food Sci. Technol. 2016, 53, 3522-3531. [CrossRef]

34. Kilic, I.H.; Sarikurkcu, C.; Karagoz, I.D.; Uren, M.C.; Kocak, M.S.; Cilkiz, M.; Tepe, B. A significant by-product of the industrial processing of pistachios: Shell skin-RP-HPLC analysis, and antioxidant and enzyme inhibitory activities of the methanol extracts of Pistacia vera L. shell skins cultivated in Gaziantep, Turkey. RSC Adv. 2016, 6, 1203-1209. [CrossRef]

35. Zhou, Y.; Zheng, J.; Li, Y.; Xu, D.-P.; Li, S.; Chen, Y.-M.; Li, H.-B. Natural Polyphenols for Prevention and Treatment of Cancer. Nutrients 2016, 8, 515. [CrossRef]

36. Lee, S.-C.; Kim, J.-H.; Jeong, S.-M.; Kim, D.-R.; Ha, J.-U.; Nam, K.C.; Ahn, D.U. Effect of far-infrared radiation on the antioxidant activity of rice hulls. J. Agric. Food Chem. 2003, 51, 4400-4403. [CrossRef]

37. Locatelli, M.; Travaglia, F.; Coïsson, J.D.; Martelli, A.; Stévigny, C.; Arlorio, M. Total antioxidant activity of hazelnut skin (Nocciola Piemonte PGI): Impact of different roasting conditions. Food Chem. 2010, 119, 1647-1655. [CrossRef]

38. Marzocchi, S.; Pasini, F.; Verardo, V.; Ciemniewska-Żytkiewicz, H.; Caboni, M.F.; Romani, S. Effects of different roasting conditions on physical-chemical properties of Polish hazelnuts (Corylus avellana L. var. Kataloński). LWT 2017, 77, 440-448. [CrossRef]

39. Lucchetti, S.; Ambra, R.; Pastore, G. Effects of peeling and/or toasting on the presence of tocopherols and phenolic compounds in four Italian hazelnut cultivars. Eur. Food Res. Technol. 2018, 244, 1057-1064. [CrossRef]

40. Schmitzer, V.; Slatnar, A.; Veberic, R.; Stampar, F.; Solar, A. Roasting affects phenolic composition and antioxidative activity of hazelnuts (Corylus avellana L.). J. Food Sci. 2011, 76, S14-S19. [CrossRef] 
41. Açar, Ö.C.; Gökmen, V.; Pellegrini, N.; Fogliano, V. Direct evaluation of the total antioxidant capacity of raw and roasted pulses, nuts and seeds. Eur. Food Res. Technol. 2009, 229, 961-969. [CrossRef]

42. Król, K.; Gantner, M.; Piotrowska, A. Morphological traits, kernel composition and sensory evaluation of hazelnut (Corylus avellana L.) cultivars grown in Poland. Agronomy 2019, 9, 703. [CrossRef]

43. Blomhoff, R.; Carlsen, M.H.; Andersen, L.F.; Jacobs, D.R., Jr. Health benefits of nuts: Potential role of antioxidants. Brit. J. 2006, 96, 52-t60. [CrossRef]

44. Shahidi, F.; Alasalvar, C.; Liyanapathirana, C.M. Antioxidant phytochemicals in hazelnut kernel (Corylus avellana L.) and hazelnut byproducts. J. Agric. Food Chem. 2007, 55, 1212-1220. [CrossRef]

45. Del Rio, D.; Calani, L.; Dall'Asta, M.; Brighenti, F. Polyphenolic composition of hazelnut skin. J. Agric. Food Chem. 2011, 59, 9935-9941. [CrossRef] [PubMed]

46. Schlörmann, W.; Birringer, M.; Böhm, V.; Löber, K.; Jahreis, G.; Lorkowski, S.; Müller, A.K.; Schöne, F.; Glei, M. Influence of roasting conditions on health-related compounds in different nuts. Food Chem. 2015, 180, 77-85. [CrossRef] [PubMed]

47. Alamprese, C.; Ratti, S.; Rossi, M. Effects of roasting conditions on hazelnut characteristics in a two-step process. J. Food Eng. 2009, 95, 272-279. [CrossRef]

(C) 2020 by the authors. Licensee MDPI, Basel, Switzerland. This article is an open access article distributed under the terms and conditions of the Creative Commons Attribution (CC BY) license (http://creativecommons.org/licenses/by/4.0/). 